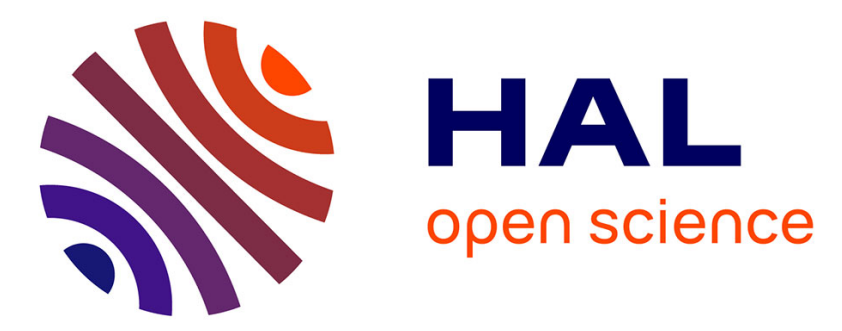

\title{
Contamination des eaux pluviales par les micropolluants: avancées du projet INOGEV
}

Johnny Gasperi, Christel Sebastian, Véronique Ruban, Mélissa Delamain,

Stéphane Percot, Laure Wiest, Cécile Mirande, Emilie Caupos, Dominique

Demare, M Diallo Kessoo, et al.

\section{To cite this version:}

Johnny Gasperi, Christel Sebastian, Véronique Ruban, Mélissa Delamain, Stéphane Percot, et al.. Contamination des eaux pluviales par les micropolluants: avancées du projet INOGEV. Techniques Sciences Méthodes , 2017, 7/8, pp.51-66. 10.1051/tsm/201778051 . hal-01581007

\section{HAL Id: hal-01581007 \\ https://hal.science/hal-01581007}

Submitted on 23 May 2018

HAL is a multi-disciplinary open access archive for the deposit and dissemination of scientific research documents, whether they are published or not. The documents may come from teaching and research institutions in France or abroad, or from public or private research centers.
L'archive ouverte pluridisciplinaire HAL, est destinée au dépôt et à la diffusion de documents scientifiques de niveau recherche, publiés ou non, émanant des établissements d'enseignement et de recherche français ou étrangers, des laboratoires publics ou privés. 


\begin{tabular}{|c|c|}
\hline 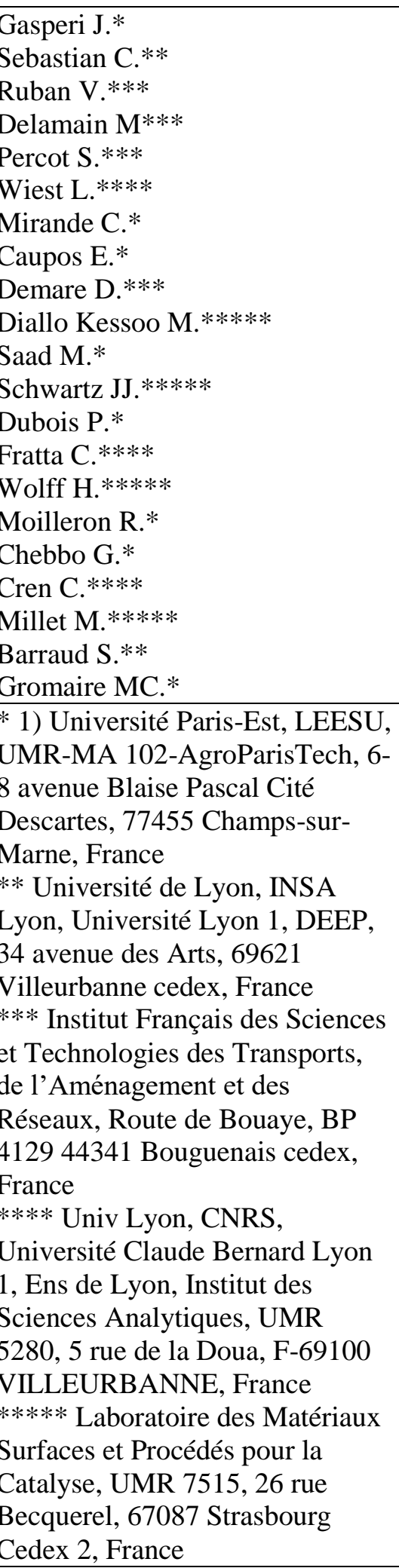 & $\begin{array}{l}\text { Contamination des eaux } \\
\text { pluviales par les } \\
\text { micropolluants : avancées } \\
\text { du projet INOGEV }\end{array}$ \\
\hline
\end{tabular}




\section{RESUME}

Cet article synthétise les résultats du projet INOGEV sur les concentrations de micropolluants organiques et métalliques dans les eaux pluviales strictes. Ce projet a permis non seulement d'étudier certains polluants jusqu'à lors faiblement documentés dans la littérature mais également d'établir les ordres de grandeurs des concentrations totales, dissoutes et particulaires dans les eaux pluviales. Excepté pour quelques micropolluants pour lesquels le trafic automobile est une source importante de contamination (certains métaux, HAP) et les PBDE, aucune différence significative n'a été observée entre les trois bassins versants étudiés, la variabilité inter-événementielle des concentrations sur un même site étant du même ordre de grandeur que la variabilité inter-sites. De manière générale, la distribution des polluants entre phases dissoute et particulaire dépend des propriétés physico-chimiques des molécules. Les métaux sont préférentiellement associés aux particules :) > 50\% for As, Cd, Mo, Ni, V, Cu et $\mathrm{Zn}$; et $>80 \%$ for $\mathrm{Co}, \mathrm{Cr}$, Pb et Ti. Pour les composés organiques, cette répartition dépend fortement du coefficient octanol - eau (Kow). Au regard des processus de production et de transfert sur les surfaces urbaines, les résultats indiquent pour un large panel de molécules que les apports atmosphériques ne contribuent que minoritairement à la pollution observée aux exutoires, leur contribution n'excédant généralement pas 30\%. Démontré pour les métaux et les HAP à travers des études antérieures, ce résultat est innovant pour les autres molécules étudiées et vient confirmer une production locale importante.

\section{MOTS-CLES}

Eaux pluviales, micropolluants ; alkylphénols ; PBDE ; métaux ; bassins versants urbains ; atmosphère 


\section{INTRODUCTION}

Depuis les années 1980, de nombreuses études ont démontré que les eaux pluviales urbaines contribuent à la détérioration de la qualité des milieux récepteurs (Brombach et al. 2005, Burton et Pitt 2002, Clark et al. 2007). Les premières investigations sur les eaux pluviales ont porté sur les paramètres globaux et les nutriments, ainsi que sur les polluants classiques tels que les métaux lourds et les hydrocarbures aromatiques polycycliques (HAP) (Brown et Peake 2006). Pour ces paramètres, des bases de données nationales et internationales ont été créées : NURP (Smullen et al. 1999) et NSQD (Pitt et Maestre 2005) aux Etats-Unis, QASTOR (Saget 1994) en France ou encore ATV (Fuchs et al. 2004) en Allemagne.

Plus récemment, la contamination des eaux pluviales par une large gamme de polluants organiques a été mise en évidence. Un grand nombre de composés (plus de 650 identifiés) sont en effet présents à l'état de traces (Barbosa et al. 2012). Parmi ces polluants, on retrouve de nombreux pesticides (Muller et al. 2002), des perturbateurs endocriniens tels que les phtalates, les alkylphénols et le bisphénol A (Björklund et al. 2009) et des polluants prioritaires de la directive cadre européenne sur l'eau (Eriksson et al. 2007, Lamprea et Ruban 2011a, Lamprea et Ruban 2011b, Zgheib et al. 2012). Afin d'élaborer des stratégies qui limitent l'impact des contaminants déversés par les eaux pluviales à divers niveaux de décision (national, régional et local), une connaissance plus fine des sources et des concentrations de ces contaminants est nécessaire. Une meilleure compréhension des processus de production et de transfert de l'atmosphère à l'exutoire des bassins est également requise. Dans le passé, des relations entre les quantités de polluants accumulés, l'imperméabilisation du bassin, le plan d'occupation des sols (Burton et Pitt 2002) et le type de drainage (Brombach et al. 2005) ont été établies pour certains polluants (métaux et HAP typiquement), mais jamais testées pour ces nouveaux contaminants. Pour quelques éléments, Smullen et al. (1999) ont montré de fortes variations de concentrations entre les résultats de trois bases de données nationales américaines (NURP, USGS et NPDES) suggérant que la qualité des eaux pluviales est susceptible de varier fortement selon les bassins versants. Sur la base de ces résultats, les auteurs incitent à ce que les futurs travaux s'attachent à caractériser plus finement les eaux pluviales à l'échelle de différents bassins versants. Rossi, en 1998, a montré que la variabilité inter-évènementielle sur un même bassin versant était si élevée qu'aucune conclusion claire ne pouvait être tirée sur une potentielle variabilité spatiale ou sur l'influence du plan d'occupation des sols sur la qualité des eaux pluviales (Rossi 1998). A ce jour, cette information reste complètement méconnue pour de nombreux polluants. Les concentrations, les flux et dans une plus large mesure, leur hétérogénéité et/ou leur homogénéité d'un site à l'autre n'ont pas été examinées.

En France, à partir de 2010, diverses études sur les micropolluants dans les eaux pluviales ont été réalisées sur les trois observatoires d'hydrologie urbaine nationaux : OTHU à Lyon (Observatoire de Terrain en Hydrologie Urbaine), OPUR à Paris (Observatoire des Polluants Urbains), et ONEVU à Nantes (Observatoire Nantais des Environnements Urbains). Sur chaque observatoire, une attention particulière a été accordée aux polluants prioritaires et certains métaux (Becouze-Lareure 2010, Bressy et al. 2011, Bressy et al. 2012, Gasperi et al. 2012, Lamprea et Ruban 2011a, Zgheib et al. 2012). En raison de différences méthodologiques (choix des polluants ciblés, procédures d'échantillonnage, méthodes analytiques, etc.), la comparaison entre les différents bassins versants ne permet pas de mettre en évidence de différence d'un site à l'autre au regard de la qualité des eaux de ruissellement. Dans ce contexte, et afin de répondre à cette question, le projet INOGEV (20102013, Innovations pour une Gestion Durable de l'Eau en Ville- Connaissance et maîtrise de la contamination des eaux pluviales urbaines) a été initié par les trois observatoires sous l'égide du réseau des observatoires français en hydrologie urbaine (SOERE URBIS). Ce projet vise à améliorer les connaissances sur la contamination des eaux pluviales dans le cadre d'une approche multidisciplinaire, en harmonisant les approches scientifiques, les méthodologies de suivis (polluants, échantillonnage, méthodes analytiques) et l'interprétation des résultats à l'échelle des trois bassins versants étudiés.

Les objectifs de cet article sont 1) d'étudier pour un large panel de polluants (n=77) la qualité des eaux pluviales, en termes de fréquence de quantification et de concentrations, 2) d'évaluer si la contamination des eaux pluviales diffère à l'échelle des trois bassins versants et déterminer si les 
paramètres comme l'occupation des sols ou les activités locales peuvent expliquer ces différences, et 3) d'évaluer les contributions relatives des retombées atmosphériques.

\section{MATERIELS ET METHODES}

\subsection{Description des sites}

Trois bassins versants urbains drainés par des réseaux d'assainissement séparatifs, ont été sélectionnés : le bassin versant de Sucy-en-Brie pour l'observatoire OPUR en région parisienne, le bassin versant de Pin Sec pour l'observatoire ONEVU à Nantes et le bassin versant de Chassieu pour l'observatoire OTHU à Lyon. Ces bassins versants présentent une diversité d'occupation du sol (pavillonnaire, résidentiel avec habitat collectif et individuel, industriel) tout en étant représentatifs des zones urbaines.

Leurs surfaces s'étendent de 30 (Pin Sec) à 228 ha (Sucy) et leurs coefficients d'imperméabilisation varient entre 27 (Sucy) et 75\% (Chassieu). Chassieu est une zone industrielle, alors que les deux autres bassins sont principalement résidentiels. Sucy est un quartier résidentiel avec une majorité de petites habitations familiales individuelles et des activités commerciales et professionnelles limitées (petits espaces de ventes et de services). Le bassin versant du Pin Sec est un quartier résidentiel avec principalement des immeubles d'habitations et des maisons individuelles. Dans l'ensemble, la majorité des bâtiments et maisons date des années 1960 ou 1970. Les densités du trafic ont été respectivement évaluées à 60000,36000 et 10000 véhicules kilomètres parcourus par jour (véhicules. $\mathrm{km} \mathrm{j}^{-1}$ ) à Sucy, Chassieu et Pin Sec. Les densités du trafic normalisées à la surface imperméable sont égales à 270, 670 et 1250 véhicules. $\mathrm{km} \mathrm{j}^{-1}$ ha imp ${ }^{-1}$ à Chassieu, Pin Sec et Sucy respectivement.

Sur chaque site, les retombées atmosphériques totales (RAT) et les eaux pluviales à l'exutoire des bassins versants ont été collectées simultanément. Selon les sites, entre 7 et 24 évènements ont été échantillonnés. L'échantillonnage a été effectué sur une période de 23 mois (de juillet 2011 à mai 2013). En raison de l'important volume nécessaire pour réaliser les analyses (plus de 20 litres afin d'accumuler suffisamment de masses pour la phase particulaire), il n'a pas été possible d'analyser tous les contaminants au cours de chaque épisode pluvieux. Deux configurations d'échantillonnages ont donc été mises en place : une pour le suivi des alkylphénols et alkylphénols éthoxylés (APnEO), des polybromodiphényléthers (PBDE) et des pesticides, et une autre configuration pour les hydrocarbures aromatiques polycycliques (HAP), le glyphosate, l'acide amino-méthylphosphonique (AMPA) et les métaux. Entre 2 et 14 évènements ont été échantillonnés pour une même famille de composés sur chaque bassin versant. Les principales caractéristiques des évènements échantillonnés (précipitation en $\mathrm{mm}$ ), intensités moyenne et maximum sur 5 minutes $\left(\mathrm{I}_{\text {moy }}\right.$ et $\mathrm{I}_{\max }$ en $\mathrm{mm} \mathrm{h}^{-1}$ ), période de temps sec précédant l'évènement (TS en jours) sont présentées en détails dans Gasperi et al. (2014). Dans l'ensemble, ces évènements pluvieux sont de faibles intensités. Les hauteurs totales de précipitations $(1,2$ à $50 \mathrm{~mm})$ et leurs durées $(00: 35$ à $60: 35)$ couvrent cependant une large diversité.

Afin d'éviter des contaminations dues aux échantillonneurs ou la sorption de certains contaminants sur les flaconnages, les RAT ont été recueillies dans un collecteur en acier inoxydable pour les polluants organiques et deux collecteurs en plastique de $0,5 \mathrm{~m}^{2}$ pour les métaux, le glyphosate et l'AMPA. Les RAT ont été collectées sur la durée totale de l'évènement pluvieux étudié et incluent la période précédente de temps sec. Les collecteurs atmosphériques ont été mis en place sur des toits (Sucy, Pin Sec) et/ou à l'écart des sources locales potentielles de contamination.

A l'exutoire des bassins versants, les eaux pluviales ont été échantillonnées au moyen de préleveurs automatiques asservis au débit et équipés de tuyaux en Teflon®. Un échantillon moyen était constitué pour chaque évènement pluvieux proportionnellement aux volumes écoulés afin d'évaluer les concentrations moyennes évènementielles (CME). Les flacons en verre ont été utilisés pour la plupart des polluants organiques, alors que le flaconnage plastique permet l'échantillonnage des métaux et de trois pesticides (glyphosate, glufosinate et son métabolite l'AMPA). L'ensemble du matériel et des procédures d'échantillonnage (étapes de nettoyage, type de bouteille, etc...) ont été harmonisés d'un observatoire à l'autre, ainsi que les procédures de blanc terrain. 


\subsection{Paramètres globaux de la qualité de l'eau et polluants analysés}

Les paramètres globaux tels que les matières en suspension (MES) et le carbone organique total, dissous et particulaire (COT, COD et COP), ont été analysés pour chaque évènement pluvieux. Au total, 77 micropolluants ont été analysés, dont 14 métaux, 30 pesticides, 16 hydrocarbures aromatiques polycycliques (HAP), 9 polybromodiphényléthers (PBDE), le bisphénol A (BPA) et 7 alkylphénols (APnEO). La liste complète des molécules ciblées, les méthodes analytiques employées et les abréviations usuelles sont stipulées dans le Tableau 1.

Pour tous les échantillons, les phases dissoute et particulaire ont été analysées. Pour les composés organiques, les phases dissoute et particulaire ont été analysées séparément pour une quantification précise des contaminants (Zgheib et al. 2011b). Les métaux ont été analysés par spectrométrie par spectroscopie de masse couplée à un plasma inductif (ICP-MS) pour la majeure partie ou par spectrométrie d'émission optique (ICP-AES) pour le Zn selon les normes françaises (NF X 31-147) sur les fractions dissoute et totale. Les polluants organiques ont été analysés par chromatographie liquide ou gazeuse couplée avec un détecteur à fluorescence ou avec un spectromètre de masse simple, en tandem ou à temps de vol. Les détails analytiques sont disponibles pour les APnEO et le bisphénol A (Cladière et al. 2013), les pesticides (Schummer et al. 2012) et les PBDE (Gilbert et al. 2012). Toutes les analyses pour une classe de contaminants donnée ont été conduites par un même laboratoire de référence.

Tableau 1 : Polluants analysés et méthodes analytiques associées

\begin{tabular}{|c|c|c|}
\hline Groupes & Méthodes & Substances et abréviations \\
\hline $\begin{array}{l}\text { Métaux } \\
(n=14)\end{array}$ & $\begin{array}{l}\text { ICP-MS } \\
\text { ICP-AES }\end{array}$ & $\begin{array}{l}\text { Arsenic (As), Cadmium }(\mathrm{Cd}) \text {, Chrome }(\mathrm{Cr}) \text {, Cuivre }(\mathrm{Cu}) \text {, Nickel } \\
(\mathrm{Ni}), \text { Plomb }(\mathrm{Pb}) \text {, Zinc }(\mathrm{Zn}) \text {, Platine }(\mathrm{Pt}) \text {, Vanadium }(\mathrm{V}) \text {, Cobalt } \\
(\mathrm{Co}), \text { Molybdène }(\mathrm{Mo}), \text { Strontium }(\mathrm{Sr}) \text {, Baryum }(\mathrm{Ba}) \text {, Titan }(\mathrm{Ti})\end{array}$ \\
\hline $\begin{array}{l}\text { HAP } \\
(n=16)\end{array}$ & GC-Tof & $\begin{array}{l}\text { Naphtalène }(\mathrm{N}) \text {, Acénaphtylène }(\mathrm{Acyl}) \text {, Acénaphtène }(\text { Acen }) \text {, } \\
\text { Fluorène }(\mathrm{F}), \text { Phénanthrène }(\mathrm{P}) \text {, Anthracène }(\mathrm{A}) \text {, Fluoranthène } \\
\text { (Fluo) } \\
\text { Pyrène }(\mathrm{Pyr}) \text {, Benzo(a)anthracène }(\mathrm{BaA}) \text {, Chrysène }(\mathrm{Chry}), \\
\text { Benzo(b)fluoranthène }(\mathrm{BbF}), \text { Benzo(k)fluoranthène }(\mathrm{BkF}), \\
\text { benzo(a)pyrène }(\mathrm{BaP}) \text {, Indéno(cd)pyrène }(\mathrm{IP}) \text {, } \\
\text { Dibenzo(ah)anthracène (DahA), Benzo(ghi)pérylène }(\mathrm{B}(\mathrm{ghi}) \mathrm{P})\end{array}$ \\
\hline $\begin{array}{l}\text { Pesticides } \\
(\mathrm{n}=30)\end{array}$ & $\begin{array}{l}\text { GC-MS } \\
\text { LC-MSMS } \\
\text { LC-Fluo }\end{array}$ & $\begin{array}{l}\text { Métaldéhyde, Glyphosate, acide amino-méthyl-phosphonique } \\
\text { (AMPA), Glufosinate, Chlorfenviphos, Diuron, Endosulfan A, } \\
\text { Folpel, Isoproturon, Aldrine, Dieldrine, Isodrine, Mécoprop, acide } \\
\text { dichlorophenoxyacétique (2,4-D), acide 4-chloro-2- } \\
\text { méthylphenoxyacétique (2,4-MCPA), Trichlopyr, Carbendazime, } \\
\text { Tsothiazolinone, Irgarol 1051, Terbutryne, Acétochlore, } \\
\text { Métolachlore, Pendimethaline, Epoxiconazole, Tébuconazole, } \\
\text { Fenpropidine, Chlorothalonil, Métazachlore, Diflufénicanil, } \\
\text { Deltaméthrine }\end{array}$ \\
\hline $\begin{array}{l}\text { PBDE } \\
(n=9)\end{array}$ & GC-MS & $\begin{array}{l}\text { BDE-28 [tri], BDE-47 [tétra], BDE-99 [penta], BDE-100 [penta], } \\
\text { BDE-153 [hexa], BDE-154 [hexa], BDE-183 [hepta], BDE-205 } \\
\text { [octa], BDE-209 [déca] }\end{array}$ \\
\hline $\begin{array}{l}\text { BPA et } \\
\text { APnEO } \\
(n=7)\end{array}$ & LC-MSMS & $\begin{array}{l}\text { Bisphénol A (BPA) } \\
\text { Nonylphénol (NP), Nonylphénol monoéthoxylate (NP1EO), } \\
\text { Nonylphénol diéthoxylate (NP2EO), Nonylphénol } \\
\text { monocarboxylate (NP1EC), 4-tert-octylphénol (OP), Octylphénol } \\
\text { monoéthoxylate (OP1EO), octylphénol diéthoxylate (OP2EO) }\end{array}$ \\
\hline \multicolumn{3}{|c|}{$\begin{array}{l}\text { Méthodes analytiques : ICP-MS=spectrométrie de masse couplée à un plasma inductif, ICP- } \\
\text { AES=spectroscopie d'émission atomique couplée à un plasma inductif, GC-MS=chromatographie gaz couplée } \\
\grave{a} \text { un spectromètre de masse, GC-Tof=chromatographie gaz couplée à un spectromètre de masse à temps de } \\
\text { vol, LC-fluo=chromatographie liquide couplée à un détecteur fluorescence, LC-MSMS=chromatographie } \\
\text { liquide couplée à un spectromètre de masse en tandem. }\end{array}$} \\
\hline
\end{tabular}


Comparativement aux incertitudes analytiques, les blancs terrains ne permettent pas de mettre en évidence une contamination des dispositifs d'échantillonnage et des procédures de prétraitement pour la plupart des polluants sur les concentrations dissoutes. Pour les concentrations totales et particulaires, des difficultés sur l'homogénéisation des échantillons ont été rencontrées et les tests menés ne permettent pas de confirmer ou d'infirmer une contamination de cette phase.

\subsection{Méthodologie d'interprétation des résultats}

Afin d'évaluer la représentativité des eaux pluviales collectées dans le cadre du projet INOGEV, la première partie de ce article est consacrée aux paramètres globaux. Les concentrations moyennes événementielles (CME) sont comparées dans un premier temps entre les sites d'étude puis avec les données issues de la littérature. Pour comparer les sites, la distribution statistique des CME est évaluée. Selon Smullen et al. (1999), les CME suivent le plus souvent une distribution log-normale. Dans cette étude, les distributions log-normale ont donc été testées (test de Shapiro-Wilk, $\alpha=0,05$ ). Puis, la moyenne et l'écart-type (ET) des CME (distribution estimée) ont été calculés en espace log et ensuite transformés dans un espace arithmétique (US-EPA 1989).

Dans la deuxième partie, les niveaux de concentrations sont examinés. En s'appuyant sur une méthodologie identique, la distribution statistique des CME de chaque polluant est évaluée (test de Shapiro-Wilk, $\alpha=0,05$ ) et les différences entre sites sont estimées (test de Kruskal-Wallis, $\alpha=0,05$ ). Pour les polluants montrant des différences d'un site à l'autre, les concentrations individuelles sur chaque site seront présentées. Lorsqu'aucune différence n'a été identifiée, les données des trois sites seront regroupées et les paramètres statistiques globaux seront fournis. La dernière partie présente la répartition des polluants entre les phases dissoutes et particulaires, ainsi que les contributions des retombées atmosphériques totales à la contamination des eaux pluviales.

\section{RESULTATS ET DISCUSSION}

\subsection{Paramètres globaux}

Les concentrations en MES, COD et COP à l'exutoire des bassins versants sont présentées sur la Figure 1. Sur chaque site et pour les trois paramètres, les CME suivent des distributions log-normales (test de Shapiro-Wilk, $\alpha=0,05$ ) et aucune différence significative pour ces paramètres n'est observée entre les trois bassins (test de Kruskal-Wallis, $\alpha=0,05$ ). Les concentrations en MES sont en accord avec celles rapportées sur les mêmes sites lors d'études précédentes (Lamprea et Ruban 2011b, Zgheib et al. 2011b). Sur Chassieu, la mesure en continu de la turbidité entre 2004 et 2011 a permis d'estimer une concentration moyenne en MES égale à $75 \mathrm{mg} \mathrm{L}^{-1}$ (Metadier et Bertrand-Krajewski 2012). Globalement, les concentrations moyennes observées sur les trois bassins versants $(148,129$ et $102 \mathrm{mg} \mathrm{L}^{-1}$ ) concordent avec celles typiquement rapportées pour les eaux pluviales dans la littérature. Les CME sont relativement proches des valeurs allemandes fournies par Fuchs et al. (2004), avec une moyenne en MES à $150 \mathrm{mg} \mathrm{L}^{-1}$ (265 sites). De même, les CME sont globalement du même ordre de grandeur que celles répertoriées dans la base de données américaine NURP (Smullen et al. 1999), avec des concentrations moyennes et médianes respectivement égales à 174 et $113 \mathrm{mg} \mathrm{L}^{-1}$. A l'échelle nationale, les concentrations mesurées sur les sites INOGEV apparaissent beaucoup plus faibles que celles précédemment rapportées en France par Saget (1994) entre 170 et $550 \mathrm{mg} \mathrm{L}^{-1}$ (médiane $420 \mathrm{mg} \mathrm{L}^{-1}$ ) sur les bassins versants parisiens (base de données QASTOR). Ces différences pourraient être le témoin de nettes améliorations des rejets d'eaux usées au sein des réseaux séparatifs. Depuis 1994, des efforts considérables ont été en effet consentis à l'amélioration de ces mauvaises connexions. 

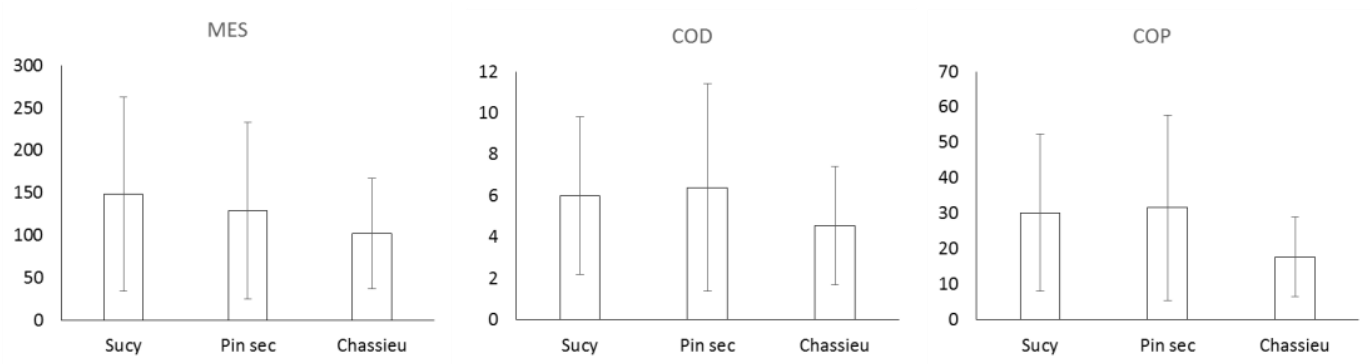

Figure 1 : Concentrations (moyennes \pm ET, en $\mathrm{mg} \mathrm{L}^{-1}$ ) pour les eaux pluviales pour Sucy $(n=24)$, Pin Sec $(n=18)$ et Chassieu $(n=7)$

\subsection{Micropolluants dans les retombées atmosphériques et à l'exutoire des bassins versants}

\section{Fréquence de quantification dans les RAT et à l'exutoire des bassins versants}

Des 77 polluants suivis, entre 42 et 48 substances (dont les métaux, les HAP, les PBDE, les APnEO et le BPA) ont été systématiquement détectées alors que 20 à 25 substances présentaient des fréquences de quantifications inférieures à $25 \%$. Dans l'ensemble, les profils de contamination sont assez homogènes entre les 3 sites, à l'exception de quelques pesticides ou des composés quantifiés à de très faibles niveaux.

Sur les 14 métaux mesurés, presque tous ont été systématiquement détectés dans les RAT et les eaux pluviales aux exutoires des bassins versants, sauf Co, Mo et Pt pour lesquels les niveaux sont inférieurs à leurs limites de détection $\left(0,6 \mu \mathrm{g} \cdot \mathrm{l}^{-1}\right.$ pour Co et $\mathrm{Mo}, 0,01 \mu \mathrm{g} \cdot \mathrm{l}^{-1}$ pour $\left.\mathrm{Pt}\right)$. Aucune différence significative n'apparait entre les sites.

Pour les HAP, 6 composés (N, Acen, F, P, Fluo et Pyr) ont été systématiquement observés dans les RAT et les eaux pluviales quel que soit le site considéré. Les autres composés correspondant pour la plupart à des HAP de haut poids moléculaire (HAP HPM, soit 5-6 cycles aromatiques) ont été détectés moins fréquemment mais leurs fréquences de quantification varient cependant entre $<20 \%$ et $60 \%$ aux exutoires des bassins. Les distributions en HAP sont similaires d'un épisode pluvieux à l'autre et d'un site à l'autre. Alors que les RAT sont caractérisées par la prédominance de HAP de faible poids moléculaire (HAP FPM, 2-4 cycles aromatiques), les eaux pluviales présentent une plus forte proportion de HAP HPM. Cette différence résulte des émissions locales sur les surfaces urbaines liées au trafic automobile. Ces émissions peuvent résulter de processus pyrolytiques (gaz d'échappement) ou de sources pétrolières (pneus en caoutchouc, fuites d'huile, matériaux bitumineux (Yunker et al. 1999)). Les signatures HAP reflètent ce mélange de contamination pyrolytique et pétrogénique (Soclo et al. 2000).

Sur les 30 pesticides recherchés , 19 composés dont 5 herbicides (métazachlore, terbutryne, pendiméthaline, trichlopyr et acétochlore), 5 fongicides (folpel, époxiconazole, fenpropidine, le chlorothalonil et le tébuconazole), 6 insecticides (chlorfenviphos, l'endosulfan, l'aldrine, la dieldrine, isodrine et deltaméthrine) et 3 algicides / molluscicides (isothiazolinone, Irgarol 1051 et métaldéhyde) n'ont jamais été détectés dans les eaux pluviales ou avec une fréquence de quantification inférieure à $20 \%$, quel que soit le bassin considéré. Les limites de détection de la plupart de ces composés varient entre 2 et $7 \mathrm{ng} \mathrm{L^{-1 }}$. L'absence de certains composés dans les eaux pluviales peut s'expliquer par leur interdiction d'utilisation en France depuis de nombreuses années. Même si cela est à relativiser, l'aldrine ou la dieldrine peuvent être citées en exemple. L'aldrine est interdit depuis 1994 mais l'étude sur l'efficacité d'un bassin de retenue montre qu'il est présent dans les sédiments apportés par les eaux de pluie. La terbutryne, l'irgarol 1051 et l'isothiazolinone n'ont pas non plus été détectés (Burkhardt et al. 2011). Ces molécules avaient été observées par Burkhardt et al. (2011) dans les eaux pluviales de zones de construction récentes, et attribuées au lessivage de matériaux de construction contenant des agents biocides. Ce type de matériau étant d'un usage récent, il est probablement peu présent que les bassins versants étudiés. 
En fait, 7 herbicides (glyphosate, glufosinate et son produit de dégradation AMPA, diuron, isoproturon, mécoprop et le 2,4- MCPA) et un fongicide (carbendazime) ont été fréquemment observés dans les eaux pluviales, indépendamment du site. En général, ces composés présentaient des taux de quantification variant entre $20 \%$ et $100 \%$ dans les eaux de ruissellement (mécoprop 050\%; isoproturon 29-100\%; 2,4-MCPA 29-75\%). Une tendance similaire est observée dans les RAT. Pour ces pesticides, des fréquences de quantifications légèrement supérieures ont été observées sur les bassins versants les plus vastes (Sucy et Chassieu), comparativement à celui du Pin Sec. Étant donné que la présence de pesticides est en grande partie dépendante du site et des activités générées sur ce site, cette légère différence pourrait suggérer que l'utilisation de pesticides aurait tendance à être plus limitée mais aussi plus spécifique sur les petits bassins versants. Ce constat peut également refléter la nouvelle politique mise en œuvre dans la région nantaise, y compris sur le bassin versant du Pin Sec, visant une réduction drastique de l'utilisation des pesticides sur les espaces publics. Sur Chassieu, qui présente à l'heure actuelle la politique la moins stricte en termes de réduction des pesticides, on note des concentrations plutôt dans la fourchette haute des concentrations observées dans les RAT et les eaux pluviales. Le diuron et le glyphosate sont utilisés comme herbicides et leur présence dans les eaux pluviales peuvent s'expliquer par une large gamme d'applications sur différents types de surfaces urbaines (Blanchoud et al. 2004, Botta et al. 2009, Kolpin et al. 2006). A l'échelle de l'agglomération parisienne et avant 2008, le diuron représentait $31 \%$ des pesticides utilisés en milieu urbain (Blanchoud et al. 2007). À l'heure actuelle, en dépit de son interdiction récente en France (décembre 2008) dans les produits phytopharmaceutiques, le diuron reste utilisé comme biocide dans certaines peintures et crépis (Burkhardt 2006). Malgré une politique incitative des autorités locales pour limiter l'utilisation de pesticides, une enquête récente menée sur le bassin du Pin Sec (Delamain et al., 2016) révèle que le glyphosate reste utilisé par les jardiniers amateurs mais là aussi on observe une diminution de son utilisation depuis quelques années. Blanchoud et al. (2007) ont estimé que les coefficients de transfert (c'est à dire le rapport entre la quantité de polluants à l'exutoire du bassin versant et la quantité de polluants appliquée sur ce bassin) atteignent respectivement $60 \%$ pour le diuron et $25 \%$ pour le glyphosate. La carbendazime peut être lessivée des nouvelles peintures et crépis antisalissure (Burkhardt et al. 2007). Le mécoprop et le 2,4-MCPA sont principalement utilisés dans les jardins, les parcs et la maintenance ferroviaire.

Pour les PBDE et sur les 9 congénères analysés, 5 PBDE (BDE-28, 47, 99, 100 et 209) ont été très fréquemment détectés contrairement aux autres congénères (BDE-153, 154, 183 et 205). Ceci est en accord avec les profils PBDE recensés dans la littérature et correspond par ailleurs aux mélanges commerciaux penta-, octa- et déca-mix (Hites 2004). En raison des préoccupations croissantes sur la santé humaine et l'environnement, le penta- et l'octa-BDE et plus récemment le déca-BDE ont été interdits en Europe. La présence de PBDE dans les RAT a été rapportée (Muresan et al. 2010, Tlili et al. 2012). Muresan et al. (2010) indiquent que les BDE-47, BDE-99 et BDE-209 contribuent à hauteur de $50 \%$ à $80 \%$ du flux atmosphérique total. Cependant à ce jour, aucune étude ne s'est attachée à analyser les PBDE dans les eaux de ruissellement. Leur présence était néanmoins attendue puisque les PBDE sont incorporés à certains matériaux de construction, pièces automobiles, plastiques ou matériel électronique (Hites 2004) et peuvent donc être relargués dans les eaux pluviales.

Enfin, le BPA et les APnEO ont été systématiquement observés dans les eaux pluviales et les RAT. Dans cet article, le terme alkylphénols fait référence à 7 composés : le nonylphénol et les nonylphénols mono- et di-éthoxylates (NP, NP1EO et NP2EO), l'octylphénol et les octylphénols mono- et di-éthoxylates (OP, OP1EO et OP2EO) et le nonylphénol monocarboxylate (NP1EC). Les nonylphénols éthoxylés (NPnEO, 80\%) et les octylphénols éthoxylés (OPnEO, 20\%) sont utilisés dans une large gamme d'applications industrielles et domestiques, tels que les lubrifiants, les détergents et les agents antistatiques (Ying et al. 2002). La présence de NP et OP dans les eaux pluviales était suspectée puisque les deux composés sont utilisés dans les peintures, certains bétons ou matériaux de construction, ou encore dans l'asphalte et certaines pièces automobiles (European Chemicals Bureau 2002). Le NP1EC, qui est un produit de dégradation des NPnEO, est aussi fréquemment identifié dans ces deux matrices. Le BPA est principalement utilisé comme monomère dans la fabrication de plastiques en polycarbonates, réputés pour leur grande résistance aux chocs et à la température (par exemple, les vitrages en plastique, les pare-chocs de voiture, etc.), ainsi que dans les résines époxy (Staples et al. 1998). Le BPA est également un adjuvant introduit au cours de la production de PVC, vernis et peintures et dans la formulation de certains produits automobiles 
(fluides de freinage, pneus). Sa présence dans les RAT (Cladière et al. 2013) et les eaux de ruissellement (Kalmykova et al. 2013) a été récemment documentée.

\section{Concentration de polluants à l'exutoire des bassins versants}

Les concentrations (moyennes \pm ET) des CME sont reportées dans le Tableau 2 pour les polluants présentant des différences d'un site à l'autre et dans le Tableau 3 pour les polluants n'en présentant pas. Pour cette dernière catégorie, les quantiles 20 et 80 (Q20 et Q80) sont également donnés.

Tableau 2 : Concentrations des polluants (moyennes \pm ET) dans les eaux pluviales présentant des différences d'un site à l'autre

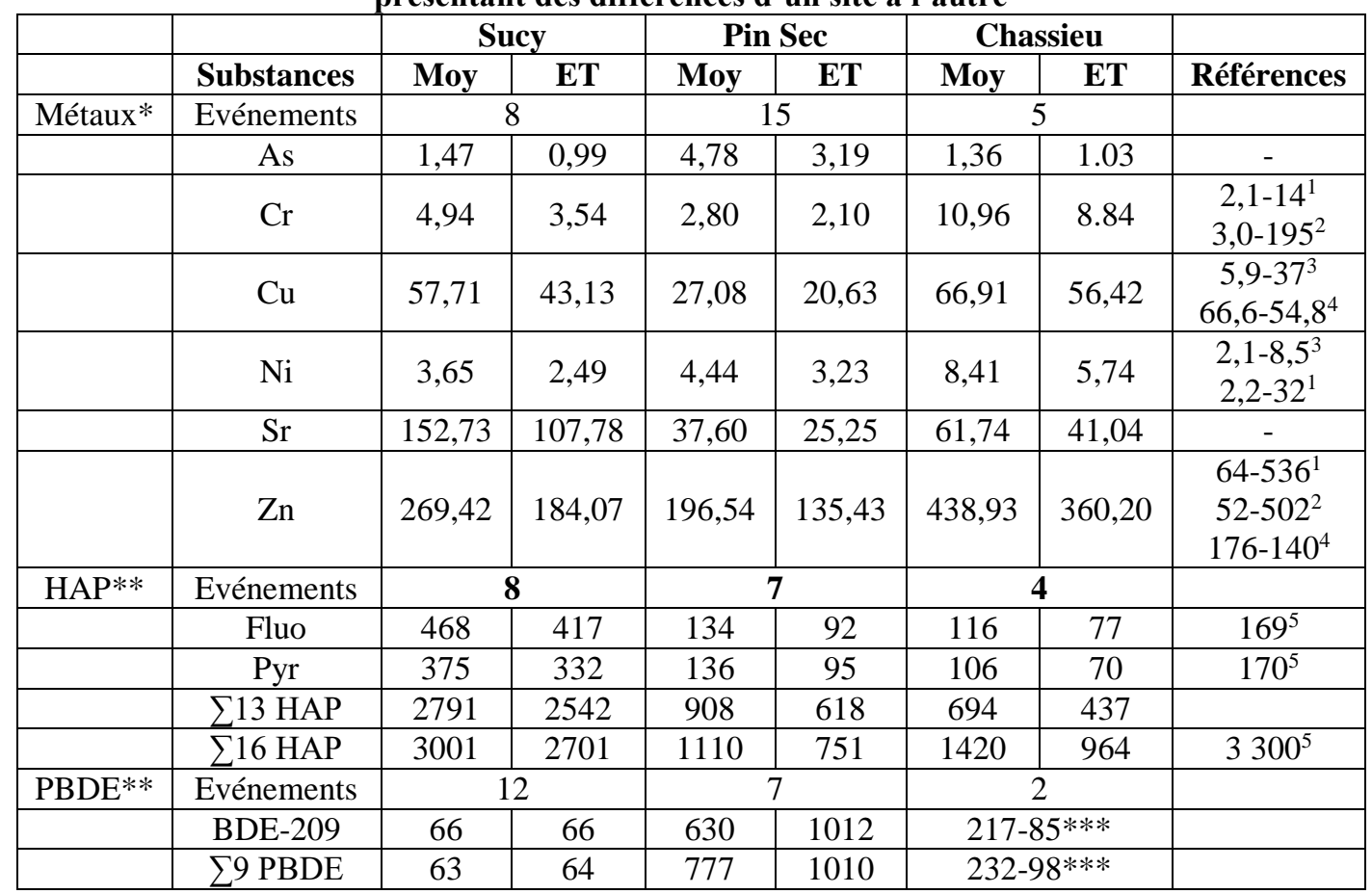

1) Lamprea et al. (2011a), 2) Rossi (1998), 3) Sabin et al. (2005) 4) base de données NURP, valeurs moyennes et médianes, 5) Zgheib et al. (2012), Valeurs médianes, *) Concentrations métalliques en $\mu g L^{-1}$,**) Concentrations en $n g L^{-1}$ pour les polluants organiques, ***) Seulement 2 évènements collectés

- Métaux

Les CME des métaux varient d'un ou plusieurs ordres de grandeur d'un échantillon à l'autre. Ces valeurs sont dans la même gamme de concentration que les mesures précédentes sur ces sites (Becouze-Lareure 2010, Lamprea et Ruban 2011b, Percot 2012), mais plutôt dans la fourchette basse des données publiées dans la littérature (Rossi 1998, Sabin et al. 2005). Le projet INOGEV fournit des informations relativement nouvelles pour des métaux peu voire pas documentés comme As, Co, Mo, Pt, Sr, Ti et V. Pour le Mo (1-12 $\mu \mathrm{g} .1^{-1}$, Q20 et Q80), Co (1-3,5 $\left.\mu \mathrm{g} . \mathrm{l}^{-1}\right), \mathrm{Pb}\left(7-35 \mu \mathrm{g} .1^{-1}\right)$, V (3-7 $\left.\mu \mathrm{g} . \mathrm{l}^{-1}\right)$, Ti $\left(10-37 \mu \mathrm{g} . \mathrm{l}^{-1}\right)$ et $\mathrm{Cd}\left(0,12\right.$ à $\left.0,42 \mu \mathrm{g} . \mathrm{l}^{-1}\right)$, nos résultats ne montrent pas de différence d'un site à l'autre à l'échelle des trois bassins versants étudiés (test de Shapiro-Wilk, $\alpha=0,05$ ). Pour As, $\mathrm{Cu}, \mathrm{Cr}, \mathrm{Ni}, \mathrm{Zn}$ et $\mathrm{Sr}$, des différences entre les sites ont été relevées (test de Shapiro-Wilk, $\alpha=0,05$ ) et les concentrations pour chaque site sont précisées dans le Tableau 2. Des concentrations plus élevées en $\mathrm{Cr}$ et $\mathrm{Ni}$ ont été mesurées à Chassieu, probablement en raison des activités industrielles locales. Des fortes concentrations en $\mathrm{Cu}, \mathrm{Zn}, \mathrm{Sr}$ et Ti ont été mesurées à Sucy. Ces métaux étant reconnus comme des traceurs du trafic automobile puisqu'ils peuvent provenir des garnitures de freins et des pneumatiques (Sternbeck et al. 2002, Thorpe et Harrison 2008), ceci suggère que les différences entre les sites pourraient être liées à des différences de densité de trafic. Les premières estimations révèlent une densité de trafic nettement plus élevée à Sucy (1 250 véhicules.km. $\mathrm{j}^{-1}$.ha $\mathrm{imp}^{-1}$ ) qu'à Chassieu ou Pin Sec (270 et 670 véhicules. $\mathrm{km} \mathrm{j}^{-1}$.ha imp $\left.{ }^{-1}\right)$. La contamination en $\mathrm{Zn}$ peut 
également être attribuée à la lixiviation de toitures, de gouttières, mobilier urbain en zinc ou en acier galvanisé (Robert-Sainte et al. 2007).

Tableau 3 : Concentrations des polluants (moyennes \pm ET, Q20 et Q80) dans les eaux pluviales ne présentant aucune différence d'un site à l'autre

\begin{tabular}{|c|c|c|c|c|c|c|}
\hline & & Moy & ET & Q20 & Q80 & Références \\
\hline Métaux* & $\mathrm{Cd}$ & 0,32 & 0,31 & 0,11 & 0,39 & $0,5-2,2^{1}$ \\
\hline \multirow[t]{5}{*}{$(n=28)$} & $\mathrm{Co}$ & 3,45 & 3,13 & 1,00 & 3,68 & - \\
\hline & Mo & 7,68 & 13,09 & 1,10 & 12,05 & - \\
\hline & $\mathrm{Pb}$ & 21,52 & 20,73 & 6,79 & 33,22 & $175-131^{2}$ \\
\hline & $\mathrm{Ti}$ & 27,80 & 28,60 & 9,70 & 37,50 & \\
\hline & $\mathrm{V}$ & 4,86 & 2,84 & 2,55 & 6,79 & \\
\hline$\underset{* * *}{\text { Pesticides }}{ }^{* *}$, & Glyphosate & 337 & 806 & 95 & 198 & \\
\hline \multirow[t]{6}{*}{$(n=19)$} & Glufosinate & 756 & 10121 & 6 & 389 & \\
\hline & AMPA & 824 & 7077 & 16 & 469 & \\
\hline & Diuron & 1213 & 10784 & 25 & 795 & \\
\hline & Isoproturon & 88 & 929 & 3 & 53 & \\
\hline & Carbendazime & 213 & 1355 & 7 & 195 & \\
\hline & Mécoprop & 3 & 7 & 1 & 2 & \\
\hline APnEO/BPA $* * *$ & BPA & 552 & 510 & 207 & 817 & $<$ LOD-107 $000^{3}$ \\
\hline \multirow[t]{7}{*}{$(n=21)$} & $\mathrm{OP}$ & 61 & 37 & 35 & 72 & \\
\hline & OP1EO & 23 & 25 & 9 & 22 & \\
\hline & OP2EO & 10 & 11 & 4 & 14 & \\
\hline & NP & 359 & 228 & 187 & 509 & $\begin{array}{c}<\text { LOQ-7 300 } \\
160-920^{4}\end{array}$ \\
\hline & NP1EO & 347 & 543 & 69 & 428 & \\
\hline & NP2EO & 164 & 216 & 52 & 141 & \\
\hline & NP1EC & 466 & 1179 & 160 & 324 & \\
\hline
\end{tabular}

1) Rossi (1998), 2) base de donnée NURP, Valeurs moyennes et médianes, 3) Kalmykova et al. (2013), 4) Bressy et al. (2012), d10-d90, *) Concentrations métalliques en $\mu \mathrm{g} \mathrm{L}{ }^{-1}$, **) Pour les pesticides, les différences d'un site à l'autre n'ont pas été testées, ***) Concentrations en ng $L^{-}$ ${ }^{1}$ pour les polluants organiques

- HAP

Pour les HAP, les concentrations totales, soit la somme des 16 HAP de l'US-EPA ( $\Sigma 16$ HAP) ou la somme des 13 HAP (hors N, Acen et Acyl) sont examinées. Alors qu'aucune différence significative n'a été trouvée pour les RAT sur les 3 sites, les analyses statistiques révèlent des différences importantes d'un site à l'autre ( $\Sigma 13$ HAP, test de Kruskal-Walli, $\alpha=0,05)$. Chassieu $\left(908 \mathrm{ng} \mathrm{L}^{-1}\right.$ pour $\Sigma 13$ HAP, tableau 3) et Pin Sec $\left(694 \mathrm{ng} \mathrm{L}^{-1}\right)$ présentent des concentrations nettement inférieures à Sucy $\left(2^{\circ} 791 \mathrm{ng} \mathrm{L}^{-1}\right)$. Les concentrations mesurées à Sucy se situent dans le même ordre de grandeur que celles précédemment rapportées par Zgheib et al. (2011a) sur ce même site ( $\Sigma 16$ HAP, 880, $3^{\circ} 300$ et $6^{\circ} 480 \mathrm{ng} \mathrm{L}^{-1}$ respectivement pour les concentrations minimums, moyennes et maximales), confirmant ainsi une forte contamination en HAP sur ce bassin par rapport aux deux autres. Même si les concentrations en MES sont comparables entre les 3 sites, les différences observées sont essentiellement liées aux teneurs en HAP des particules collectées. La teneur en HAP moyenne mesurée à Sucy (environ $\left.19^{\circ} 000 \mathrm{ng} \mathrm{g}^{-1}\right)$ dépasse nettement celles rapportées à Chassieu $\left(6^{\circ} 000 \mathrm{ng}\right.$ $\left.\mathrm{g}^{-1}\right)$ ou Pin Sec $\left(7^{\circ} 000 \mathrm{ng} \mathrm{g}^{-1}\right)$. Dans l'ensemble, les concentrations dans les eaux pluviales sont plus élevées que celles observées dans les RAT, indiquant ainsi une production locale. Aucune corrélation n'a été trouvée entre les HAP, les MES et le carbone organique dissous et particulaire (test de Spearman, $\mathrm{R}^{2}<0,3$ ). Suite probablement à un nombre limité d'évènements pluvieux, aucune corrélation saisonnière n'a été identifiée.

Comme pour certains métaux, la différence de contamination dans les eaux pluviales pourrait être attribuable à la densité du trafic automobile. Les concentrations les plus élevées ont été systématiquement trouvées à Sucy, qui présente la densité de trafic la plus importante. Le bassin industriel de Chassieu génère les concentrations de HAP les plus faibles, à l'exception de 
concentrations extrêmement élevées mais très ponctuelles de naphtalène sur quelques échantillons. Ces faibles concentrations de HAP étaient inattendues, en raison des nombreuses activités industrielles et logistiques sur ce bassin ainsi que de la proximité de la rocade lyonnaise, mais elles restent toutefois concordantes avec la faible densité de circulation au sein de ce bassin (270 véhicules.km.j ${ }^{-1}$ ha imp ${ }^{-1}$ ).

\section{- Pesticides}

Parmi les pesticides les plus couramment détectés, le glyphosate (95-198 ng L-1, Q20 et Q80), l'AMPA (16-469 $\left.\mathrm{ng} \mathrm{L}^{-1}\right)$, le diuron $\left(25-795 \mathrm{ng} \mathrm{L}^{-1}\right)$ et le glufosinate $\left(6-389 \mathrm{ng} \mathrm{L}^{-1}\right)$ sont prédominants dans les eaux pluviales. L'isoproturon (3-53 $\mathrm{ng} \mathrm{L}^{-1}$ ) et la carbendazime (7-195 $\mathrm{ng} \mathrm{L}^{-1}$ ) ont été quantifiés à de plus faibles niveaux, tandis que les concentrations de pesticides rémanents (mécoprop, 2,4-D et 2,4-MCPA) n'excèdent pas $5 \mathrm{ng} \mathrm{L}{ }^{-1}$. Etant donné le nombre limité d'évènements pluvieux (de 4 à 8 évènements selon le site), la différence de concentrations entre les sites n'a pas été testée statistiquement. Les données ont été compilées (Tableau 3). Malgré quelques études ((Blanchoud et al. 2004, Huang et al. 2004, Quaghebeur et al. 2004), la présence de ces herbicides et leur gamme de concentrations dans les eaux pluviales restent encore mal documentées. De fortes concentrations en glyphosate ont été mesurées sur le bassin du Pin Sec, où l'utilisation de ce pesticide par les services municipaux est limitée. A l'échelle des sites INOGEV, on peut raisonnablement supposer que le glyphosate continue d'être utilisé par les particuliers. Des concentrations beaucoup plus faibles en diuron et carbendazime ont été observées à Chassieu comparativement à Sucy et Pin Sec. Même si l'utilisation reste limitée à des façades relativement nouvelles ou récemment rénovées, le diuron et la carbendazime peuvent être lessivés à partir de nouvelles peintures et de crépis antisalissure à de fortes concentrations (Burkhardt et al. 2007). La plus forte proportion de bâtiments industriels à façades métalliques sur Chassieu et donc une probabilité moindre de lessivage de ces composés depuis les crépis et peintures de façades comparativement aux autres bassins, pourrait expliquer ces différences. Malgré son interdiction, le diuron pourrait encore être utilisé par les jardiniers privés ou s'être accumulé dans les sols. Des concentrations élevées d'herbicides ont été ponctuellement observées $\left(1^{\circ} 500-3^{\circ} 000 \mathrm{ng} \mathrm{L}^{-1}\right)$ indépendamment du site ou de la période d'étude. Ces fortes concentrations dépendent de différents facteurs affectant la quantité de pesticides remobilisés, tels que la durée entre l'application et les précipitations, l'imperméabilisation des surfaces traitées ou les caractéristiques des évènements pluvieux (Huang et al. 2004).

- $\quad$ PBDE

Parmi les PBDE détectés dans les eaux pluviales, le déca-BDE (BDE-209) est prédominant avec des concentrations variant de 23 à $251 \mathrm{ng} \mathrm{L}^{-1}(\mathrm{Q} 20$ et Q80) et avec une contribution relative médiane de l'ordre de $90 \%$ pour la somme des PBDE ( 59 PBDE). Les autres congénères varient globalement de 0,5 à 3,0 ng L ${ }^{-1}$. Pour les tri- à hepta-BDE, le BDE-47 et BDE-99 sont les congénères les plus présents, avec des abondances moyennes relatives de 5\% et 3\%, respectivement. Alors que la contamination par les PBDE du compartiment atmosphérique est connue (Muresan et al. 2010, ter Schure et al. 2004), aucune donnée expérimentale sur les niveaux de PBDE dans les eaux pluviales n'est disponible. Bien qu'aucune différence géographique n'ait été observée pour la contamination des RAT, des différences significatives d'un site à l'autre ont été observées pour la contamination des eaux pluviales ( $\Sigma 8$ PBDE, test de Kruskal-Wallis, $\alpha=0,05$, Tableau 2). Des concentrations plus faibles en PBDE ont été effectivement trouvées à Sucy, par rapport aux autres bassins versants. À ce jour, aucune explication ne peut être fournie pour expliciter ces différences. Quel que soit le site, les concentrations en BDE-209 à l'exutoire des bassins ont été significativement plus élevées que celles mesurées dans les RAT au cours de cette étude ( $\Sigma 8$ PBDE, 0,4 à 8,6 ng L $\mathrm{L}^{-1}$ ) ou bien dans celles rapportées en Suède pour les zones urbaines ( $\Sigma 8$ PBDE, 2,5 à 14,4 ng L-1, ter Schuré et al. 2004).

- $\quad$ Bisphénol A et APnEO

Pour le BPA et les APnEO, aucune différence entre les sites n'a été observée (test de Kruskal-Wallis, $\alpha=0,05)$. Les concentrations moyennes sont répertoriées dans le Tableau 3. Les CME moyennes du BPA et du NP ont été respectivement estimées à 552 et $359 \mathrm{ng} \mathrm{L}^{-1}$ et apparaissent nettement plus élevées que celles rapportées pour l'eau de pluie à Paris (10-180 ng L L $^{-1}$ pour le BPA et <LOD-167 ng L ${ }^{-1}$ pour le NP (Cladière et al. 2013)) mais équivalentes à celles des eaux de ruissellement et des lixiviats de décharges en Suède (<LOD-107 $000 \mathrm{ng} \mathrm{L}^{-1}$ pour le BPA et $<\mathrm{LQ}-7300 \mathrm{ng} \mathrm{L}^{-1}$ pour le NP (Kalmykova et al. 2013)). À l'échelle nationale, les niveaux de NP sont également comparables à ceux rapportés par Bressy et al. (2012), à savoir 160 à 920 ng L-1 (d10-d90, valeurs respectivement 
du premier décile d10 et du dernier décile d90). Les NP, NP1EO et NP2EO sont prédominants par rapport aux OP, OP1EO et OP2EO. Dans notre étude, le NP a tendance à présenter des concentrations plus élevées que les NP1EO et NP2EO, contrairement aux résultats suédois de Bjorklund et al. (2009). Indépendamment du site et de l'évènement pluvieux considéré, les distributions APnEO demeurent relativement homogènes et suivent l'ordre suivant: NP (42 $\pm 25 \%)$ $>\operatorname{NP} 1 E O(25 \pm 11 \%) \approx \operatorname{NP} 1 E C(21 \pm 9 \%)>\operatorname{NP} 2 E O(12 \pm 4 \%)$. Pour la première fois, la présence de NP1EC a été signalée dans les eaux de pluviales, avec des concentrations nettement supérieures à celles mesurées dans les RAT $\left(<3 \mathrm{ng} \mathrm{L}^{-1}\right)$. Une corrélation positive des NP1EO et NP2EO (test de Spearman, $\mathrm{p}=0,005$ et $0,008, \mathrm{R}^{2}=0,558$ et 0,583 ) avec le carbone organique dissous (COD) a été trouvée. Au regard de la directive cadre européenne sur l'eau, la norme de qualité environnementale (NQE) définie pour le NP (300 ng L $\left.{ }^{-1}\right)$ a souvent été dépassée même si on ne peut les comparer directement puisque les NQE sont destinées aux évaluations dans les milieux récepteurs (11 fois en 22 évènements échantillonnés).

\subsection{Répartition des polluants entre les phases dissoutes et particulaires}

Le pourcentage de polluants présents dans la phase particulaire est présenté Tableau 4. Pour tous les polluants, aucune différence significative entre les trois sites n'a été remarquée (test de KruskalWallis, $\alpha=0,05)$, suggérant ainsi que cette distribution n'est pas dépendante du site mais est liée aux propriétés physico-chimiques de la substance. Cette évaluation peut se révéler utile dans le choix du dispositif de traitement des eaux pluviales.

Tableau 4 : Pourcentage de polluants présents en phase particulaire dans les eaux pluviales

\begin{tabular}{|c|c|c|c|c|}
\hline & $<20 \%$ & $<50 \%$ & $50-80 \%$ & $>80 \%$ \\
\hline Métaux & $\operatorname{Sr}(13 \pm 10)$ & As $(48 \pm 18)$ & $\begin{array}{l}\mathrm{Cd}(63 \pm 30) \\
\mathrm{Mo}(63 \pm 40) \\
\mathrm{Ni}(54 \pm 18) \\
\mathrm{V}(62 \pm 18) \\
\mathrm{Zn}(60 \pm 23) \\
\mathrm{Cu}(73 \pm 13)\end{array}$ & $\begin{array}{l}\mathrm{Co}(80 \pm 34) \\
\mathrm{Cr}(85 \pm 11) \\
\mathrm{Pb}(94 \pm 4) \\
\mathrm{Ti}(94 \pm 9)\end{array}$ \\
\hline HAP & & $\mathrm{N}(44 \pm 28)$ & $\begin{array}{l}\mathrm{A}(60 \pm 44) \\
\mathrm{F}(70 \pm 31)\end{array}$ & $\begin{array}{c}\text { Acyl }(90 \pm 37) \\
\text { Acen }(82 \pm 28) \\
\text { P }(84 \pm 12) \\
\text { Fluo }(93 \pm 5) \\
\text { Pyr }(93 \pm 5) \\
\text { B(a)A }(100 \pm 22) \\
\text { Chry }(97 \pm 4) \\
\text { B(b)F }(99 \pm 2) \\
\text { B(k)F }(99 \pm 2) \\
\text { B(a)P }(100 \pm 1) \\
\text { IP, d(ah)A }\left(100^{*}\right) \\
\text { B(ghi)P }(99 \pm 1)\end{array}$ \\
\hline Pesticides & $\begin{array}{c}\text { Diuron }(6 \pm 41) \\
\text { Glyphosate }(14 \\
\pm 43)\end{array}$ & $\begin{array}{l}\text { Glyfosinate }(43 \pm \\
46) \\
\text { Isoproturon }(42 \pm \\
43)\end{array}$ & & \\
\hline PBDE & & & $\begin{array}{c}\text { BDE-28 }(71 \pm 33) \\
\text { BDE-100 }(55 \pm 34)\end{array}$ & $\begin{array}{l}\text { BDE-47 }(95 \pm 33) \\
\text { BDE-99 }(86 \pm 42) \\
\text { BDE-209 }(99 \pm 7)\end{array}$ \\
\hline $\begin{array}{l}\text { APnEO } \\
\text { et BPA }\end{array}$ & $\begin{array}{c}\text { BPA }(18 \pm 11) \\
\text { OP1EO }(14 \pm \\
23)\end{array}$ & $\begin{array}{c}\text { OP }(45 \pm 22) \\
\text { OP2EO }(32 \pm 16) \\
\text { NP }(40 \pm 22) \\
\text { NP1EO }(39 \pm 20) \\
\text { NP2EO }(29 \pm 17) \\
\text { NP1EC }(38 \pm 30)\end{array}$ & & \\
\hline
\end{tabular}

*) Détecté seulement dans la phase particulaire 
La plupart des métaux sont majoritairement liés à la phase particulaire (> 50\%), excepté le $\mathrm{Sr}(13 \pm$ $10 \%$ ). Cette tendance est plus fortement prononcée pour $\mathrm{Co}, \mathrm{Cr}, \mathrm{Pb}$ et $\mathrm{Ti}$, et dans une moindre mesure pour $\mathrm{Cu}$ (Tableau 4). Les autres métaux ( $\mathrm{As}, \mathrm{Cd}, \mathrm{Ni}, \mathrm{V}$, Mo et $\mathrm{Zn}$ ) présentent un comportement intermédiaire puisque la phase particulaire varie de $48 \pm 18 \%$ (As) à $63 \pm 40 \%$ (Mo). Ces distributions sont comparables à celles rapportées pour les eaux de ruissellement de chaussées (Gromaire 1998). Selon cette étude, les métaux étaient principalement liés aux particules (97\%, $83 \%, 67 \%$ et $52 \%$ pour $\mathrm{Pb}, \mathrm{Cd} \mathrm{Cu}$, et $\mathrm{Zn}$ ).

La répartition des polluants organiques entre les phases dissoute et particulaire dépend de leurs propriétés physico-chimiques. Les HAP sont préférentiellement associés aux particules, mais une différence nette entre les HAP de faible poids moléculaire (50\% à $80 \%$ des HAP totaux) et les HAP de haut poids moléculaire (> 80\%) est observable. Comme le laissaient présager leur log Kow compris entre 5,8 et 9,0, les PBDE sont très majoritairement associés aux particules. Pour ces composés, des solutions de gestion classiques basées sur la rétention et la décantation semblent être pertinentes (Pitt et al. 1995). Cette étude confirme cependant que la fraction dissoute peut être prédominante pour certains contaminants comme les pesticides, le BPA et les APnEO. Le BPA (log Kow=2,2 à 3,2), l'OP1EO (log Kow=4,1) et le NP1EC (log Kow indéterminé) ont été principalement observés dans la fraction dissoute. Pour les APnEO et en dépit d'un log Kow élevé $(4,0$ à 5,7), la fraction particulaire ne dépasse pas $45 \%$. Ceci pourrait être expliqué par les structures des APnEO (composés amphiphiles) ou encore par la non atteinte d'un équilibre dans le réseau séparatif. Dans le même esprit, le diuron ( $\log K o w=2,7)$, l'isoproturon (log Kow=2,9), le glyphosate $(\log \mathrm{Kow}=-4,0)$ et le glufosinate $(\log \mathrm{Kow}=-5,3)$ sont majoritairement sous forme dissoute, Leurs distributions entre les phases dissoutes et particulaires sont en accord avec leurs valeurs extrêmement faibles de log Kow. La répartition de l'AMPA (log Kow=-0,82) n'a pas été calculée car ce composé n'a pas été détecté dans la phase dissoute due à une limite de détection élevée (60 ng L $\mathrm{L}^{-1}$ pour l'AMPA). Bien que le log Kow ne décrive pas précisément le comportement de tous les pesticides, ce coefficient peut être utilisé comme un indicateur pour prédire la distribution des polluants entre les fractions dissoute et particulaire. D'autres paramètres peuvent également affecter la répartition, par exemple, les structures et les charges moléculaires.

\subsection{Contributions des dépôts atmosphériques à la pollution des eaux pluviales}

Pour chaque polluant, les contributions des retombées atmosphériques totales à la pollution des eaux pluviales ont été calculées. A l'échelle de l'évènement pluvieux, le rapport entre les concentrations des RAT et les concentrations des eaux pluviales a été évalué ; les moyennes \pm ET sont présentées dans Tableau 5.

Tableau 5 : Contributions (en \%) des RAT à la pollution des eaux pluviales

\begin{tabular}{|c|c|c|c|}
\hline & $\begin{array}{c}* \mathrm{C}_{[\mathrm{RAT}]} / \mathrm{C}_{\text {[exutoire] }}< \\
10 \%\end{array}$ & $\begin{array}{c}\mathrm{C}_{[\mathrm{RAT}]} / \mathrm{C}_{\text {[exutoire] }}< \\
20 \%\end{array}$ & $\begin{array}{c}\mathrm{C}_{[\mathrm{RAT}]} / \mathrm{C}_{\text {[exutoire] }}> \\
20 \%\end{array}$ \\
\hline $\begin{array}{l}\text { Paramètres } \\
\text { globaux }\end{array}$ & $\begin{array}{l}\operatorname{MES}(8 \pm 11) \\
\operatorname{COP}(9 \pm 13)\end{array}$ & & $\operatorname{COD}(25 \pm 15)$ \\
\hline \multirow[b]{2}{*}{ Métaux } & $\begin{array}{l}\text { As }(7 \pm 4) \\
\operatorname{Sr}(9 \pm 7)\end{array}$ & $\begin{array}{c}\mathrm{Ti}(13 \pm 24) \\
\mathrm{Pb}(11 \pm 12) \\
\mathrm{Cu}(20 \pm 17)\end{array}$ & $\begin{array}{l}\mathrm{Cu}(20 \pm 17) \\
\mathrm{Cr}(32 \pm 53) \\
\mathrm{Ni}(32 \pm 31) \\
\mathrm{Cd}(33 \pm 29)\end{array}$ \\
\hline & $\begin{array}{c}\mathrm{Sr} \text { Sucy }(3 \pm 1) \\
\mathrm{Sr} \text { Chassieu }(5 \pm 3) \\
\mathrm{Zn} \mathrm{n}_{\text {Sucy }}(9 \pm 5) \\
\mathrm{Cr}_{\text {Sucy }}(10 \pm 5) \\
\mathrm{Cr}_{\text {Chassieu }}(8 \pm 6)\end{array}$ & $\begin{array}{c}\operatorname{Sr}_{\text {Pin Sec }}(14 \pm 6) \\
\mathrm{Zn}_{\text {Chassieu }}(15 \pm 9)\end{array}$ & $\begin{array}{c}\mathrm{Zn} \operatorname{Pin} \operatorname{Sec}(86 \pm 127) \\
\mathrm{Cr}_{\operatorname{Pin} \operatorname{Sec}}(55 \pm 68)\end{array}$ \\
\hline HAP & $\begin{array}{c}\mathrm{B}(\mathrm{a}) \mathrm{P}(4 \pm 11) \\
\mathrm{I}(\mathrm{cd}) \mathrm{P}(1 \pm 4) \\
\mathrm{B}(\mathrm{ghi}) \mathrm{P}(4 \pm 8)\end{array}$ & $\begin{array}{c}\text { A }(13 \pm 34) \\
\text { Fluo }(17 \pm 19) \\
\text { Pyr }(14 \pm 14) \\
\text { Chry }(13 \pm 20) \\
\text { B(b)F }(14 \pm 21) \\
\text { B(k)F }(11 \pm 20) \\
\text { B(a)A }(11 \pm 15)\end{array}$ & $\begin{array}{c}\mathrm{N}(68 \pm 32) \\
\text { Acyl }(24 \pm 25) \\
\text { Acen }(40 \pm 45) \\
\mathrm{F}(28 \pm 20) \\
\mathrm{P}(26 \pm 22)\end{array}$ \\
\hline
\end{tabular}




\begin{tabular}{|c|c|c|c|}
\hline \multirow{3}{*}{ PBDE } & BDE-47 $(6 \pm 4)$ & BDE 28 $(18 \pm 24)$ & \\
& BDE-99 $(9 \pm 15)$ & BDE-100 (16 \pm 14$)$ & \\
& BDE-154 $(5 \pm 6)$ & BDE-153 (12 \pm 16$)$ & \\
& BDE-209 $(11 \pm 19)$ & \\
\hline \multirow{3}{*}{ APnEO et BPA } & NP1EC $(4 \pm 3)$ & NP $(19 \pm 16)$ & \\
& OP $(8 \pm 5)$ & NP1EO $(18 \pm 21)$ & NP2EO $(32 \pm 21)$ \\
& BPA $(4 \pm 4)$ & OP1EO $(16 \pm 21)$ & \\
\hline
\end{tabular}

$* C_{[R A T]} / C_{\text {[exutoire] }}:$ rapport entre les concentrations des retombées atmosphériques totales et les concentrations mesurées dans les eaux pluviales à l'exutoire du bassin versant

Excepté pour quelques rares substances, les contributions des RAT sont dans l'ensemble assez faibles, et ne dépassent pas 30\%. Pour les métaux et en raison des faibles concentrations trouvées sur les trois sites pour les RAT $\left(<1 \mu \mathrm{g} .1^{-1}\right.$ pour As, Cr, Ni et V; $2-4 \mu \mathrm{g} . .^{-1}$ pour Sr; 3-12 $\mu \mathrm{g} . \mathrm{l}^{-1}$ pour le $\mathrm{Cu}$ et $15-50 \mu \mathrm{g} . \mathrm{l}^{-1}$ pour $\mathrm{Zn}$ ), les RAT représentent moins de $20 \%$ de la pollution retrouvées à l'exutoire pour 6 métaux ( $\mathrm{As}, \mathrm{Pb}, \mathrm{Sr}, \mathrm{Ti}, \mathrm{V}$ et $\mathrm{Cu}$ ). Pour l'As, le $\mathrm{Sr}$ et le $\mathrm{V}$, cette contribution n'excède pas $10 \%$. Très ponctuellement et dans certains cas $(\mathrm{Cd}, \mathrm{Cr}, \mathrm{Ni})$, la contribution des RAT peut dépasser 30\%. Dans l'ensemble, les contributions des RAT aux eaux pluviales sont assez semblables à l'échelle des trois sites, à l'exception du $\mathrm{Cr}$, du $\mathrm{Sr}$ et du $\mathrm{Zn}$. Des différences ont en effet été observées pour $\mathrm{Cr}(55 \%$ au Pin Sec vs, $8 \%$ et $10 \%$ à Chassieu et Sucy), le Sr (14\% au Pin Sec vs, $5 \%$ et $3 \%$ à Chassieu et Sucy) et Zn (86\% au Pin Sec vs, $9 \%$ et $15 \%$ à Chassieu et Sucy). Une forte contribution atmosphérique en Zn a été observée sur le bassin du Pin Sec $(86 \% \pm 127 \%)$. Cette forte contribution s'explique principalement par les trois premières campagnes (décembre 2011 à mars 2012), au cours desquelles des concentrations atmosphériques inhabituellement élevées ont été mesurées (de 122 à $537 \mathrm{mg} . \mathrm{L}^{-1}$ ). Ces concentrations élevées peuvent sans doute résulter des travaux de toiture (feuille en $\mathrm{Zn}$ ) menés à proximité du dispositif d'échantillonnage, mais cette hypothèse n'a pu être vérifiée.

Pour les HAP, les PBDE, les APnEO et le BPA, les contributions atmosphériques demeurent faibles, confirmant ainsi une forte production locale. Mis à part pour les HAP et le NP, cette production n'avait pas encore été mise en évidence dans la littérature pour un si large panel de substances. En ce qui concerne les HAP, et selon la littérature, le lessivage des chaussées semble être la principale source de HAP dans les eaux pluviales urbaines (Motelay-Massei et al, 2006). Pour d'autres familles comme les APnEO et les PBDE, le lessivage des chaussées ou d'autres surfaces urbaines (toitures, bâti, etc.) ou encore des véhicules peut constituer une source de contamination puisque ces composés sont utilisés dans les matériaux de construction et de pièces automobiles. Les sources de ces composés doivent être étudiées de manière plus approfondie. 


\section{CONCLUSIONS}

Le présent article synthétise les résultats obtenus dans le cadre du projet INOGEV sur les concentrations de micropolluants dans les eaux pluviales à l'exutoire de trois bassins versants. Ce projet a permis la création d'une base de données conséquente, et permet d'améliorer notre compréhension de la pollution véhiculée par les eaux pluviales et les sources (part de l'atmosphère, part des voiries, part de certaines toitures). Pour les eaux pluviales, des connaissances nouvelles et pertinentes ont été ainsi générées pour de nombreux et nouveaux contaminants, tels que certains métaux (As, Ti, Sr, V) et pesticides, les PBDE, les APnEO et le BPA. Certains de ces polluants étaient jusqu'à lors faiblement documentés dans la littérature. Ce projet a permis non seulement de les documenter mais également d'établir les ordres de grandeurs des concentrations totales, dissoutes et particulaires dans les eaux pluviales. La base de données ainsi créée pourra être utilisée pour développer des outils d'aide à la décision.

Excepté pour quelques micropolluants pour lesquels le trafic automobile est une source importante de contamination (certains métaux, HAP) et les PBDE, ce projet ne permet pas de mettre en évidence de différences significatives entre les trois bassins, la variabilité inter-événementielle des concentrations sur un même site étant du même ordre de grandeur que la variabilité inter-sites. Ces observations confirment non seulement les conclusions tirées à l'échelle de différents bassins urbanisés parisiens, mais les renforcent dans la mesure où les trois bassins considérés sont encore plus contrastés (Zgheib et al. 2011a).

De manière générale, la distribution des polluants entre phases dissoute et particulaire ne dépend pas des sites, mais dépend des propriétés physico-chimiques des molécules. Comme souligné dans la littérature, les métaux sont préférentiellement associés aux particules : > 50\% for As, Cd, Mo, Ni, $\mathrm{V}, \mathrm{Cu}$ et $\mathrm{Zn}$; et $>80 \%$ for $\mathrm{Co}, \mathrm{Cr}, \mathrm{Pb}$ et $\mathrm{Ti}$. Pour les composés organiques, cette répartition dépend fortement du Kow. Même si ce dernier ne permet pas de prédire précisément la répartition des polluants, il demeure un bon indicateur de leur comportement.

Au regard des processus de production et de transfert, ce projet a démontré pour un large panel de molécules que les RAT ne contribuent que minoritairement à la pollution observée aux exutoires. Leur contribution n'excède généralement pas 30\%. Démontré pour les métaux et les HAP à travers des études antérieures, ce résultat est innovant pour les autres molécules et vient confirmer une production locale importante pour des molécules telles le BPA, les APnEO et les PBDE. Cette production est à relier au lessivage des surfaces urbaines, immeubles et véhicules.

En perspective, des corrélations entre groupes de polluants pourraient être recherchées afin d'identifier des traceurs. Cette recherche demeure cependant délicate dans la mesure où le nombre d'événements pluvieux diffère selon les familles et les sites et qu'au final cette investigation ne pourrait être menée que sur un nombre limité de pluie. De même, une recherche plus approfondie entre les variables explicatives des pluies et les flux de micropolluants pourrait être aussi menée. 


\section{BIBLIOGRAPHIE}

\section{GROMAIRE 1998}

Barbosa AE, Fernandes JN, David LM (2012): Key issues for sustainable urban stormwater management. Water Research 46, 6787-6798.

Becouze-Lareure C 2010: Caractérisation et estimation des flux de substances prioritaires dans les rejets urbains par temps de pluie sur deux bassins versants, PhD Thesis INSA de Lyon, 308 pp.

Becouze C, Wiest L, Baudot R, Bertrand-Krajewski J-L, Cren-Olive C (2011): Optimisation of pressurised liquid extraction for the ultra-trace quantification of 20 priority substances from the European Water Framework Directive in atmospheric particles by GC-MS and LC-FLD-MS/MS. Analytica Chimica Acta 693, 47-53.

Björklund K, Cousins AP, Strömvall A-M, Malmqvist P-A (2009): Phthalates and nonylphenols in urban runoff: Occurrence, distribution and area emission factors. Science of the Total Environment 407, 4665-4672.

Blanchoud H, Farrugia F, Mouchel JM (2004): Pesticide uses and transfers in urbanised catchments. Chemosphere 55, 905-913.

Blanchoud H, Moreau-Guigon E, Farrugia F, Chevreuil M, Mouchel JM (2007): Contribution by urban and agricultural pesticide uses to water contamination at the scale of the Marne watershed. Science of the Total Environment 375, 168-179.

Botta F, Lavison G, Couturier G, Alliot F, Moreau-Guigon E, Fauchon N, Guery B, Chevreuil M, Blanchoud H (2009): Transfer of glyphosate and its degradate AMPA to surface waters through urban sewerage systems. Chemosphere 77, 133-139.

Bressy A, Gromaire MC, Lorgeoux C, Chebbo G (2011): Alkylphenols in atmospheric depositions and urban runoff. Water Science and Technology 63, 671-679.

Bressy A, Gromaire MC, Lorgeoux C, Saad M, Leroy F, Chebbo G (2012): Towards the determination of an optimal scale for stormwater quality management: Micropollutants in a small residential catchment. Water Research 46, 6799-6810.

Brombach H, Weiss G, Fuchs S (2005): A new database on urban runoff pollution: comparison of separate and combined sewer systems. Water Science and Technology 51, 119-128.

Brown JN, Peake BM (2006): Sources of heavy metals and polycyclic aromatic hydrocarbons in urban stormwater runoff. Science of the Total Environment 359, 145-155.

Burkhardt M (2006): Einsatz von biociden in Fassaden. Applica 12, 8-14.

Burkhardt M, Kupper T, Hean S, Haag R, Schmid P, Kohler M, Boller M (2007): Biocides used in building materials and their leaching behavior to sewer systems. Water Science and Technology 56, 63-67.

Burkhardt M, Zuleeg S, Vonbank R, Schmid P, Hean S, Lamani X, Bester K, Boller M (2011): Leaching of additives from construction materials to urban storm water runoff. Water Science and Technology 63, 1974-1982.

Burton GA, Pitt R (2002): Stormwater Effects Handbook: A Toolbox for Watershed Managers Lewis Publisher, 911 pp.

Cladière M, Gasperi J, Lorgeoux C, Bonhomme C, Rocher V, Tassin B (2013): Alkylphenolic compounds and bisphenol A contamination within a heavily urbanized area: case study of Paris. Environmental Science and Pollution Research 20, 2973-2983.

Clark SE, Burian S, Pitt R, Field R (2007): Urban wet-weather flows. Water Environment Research 79, 1166-1227.

Delamain M., Ruban V., Rodriguez F. (2016). Cmparaison des pratiques d'entretien des surfaces urbaines entre le bassin versant du pin sec (Nantes) et un écoquartier adjacent - cas des pesticides et des métaux. TSM

Eriksson E, Baun A, Scholes L, Ledin A, Ahlman S, Revitt M, Noutsopoulos C, Mikkelsen PS (2007): Selected stormwater priority pollutants - a European perspective. Science of the Total Environment 383, 41-51.

European Chemicals Bureau 2002: Risk Assessment: 4-nonylphenol (branched) and nonylphenol, Rep. No. EUR 20387 EN. Office for Official Publications of the European Communities, Luxembourg.

Fuchs S, Brombach H, Weiss G (2004): New database on urban runoff pollution, Novatech, Lyon, France, pp. 145-152. 
Gasperi J, Zgheib S, Cladière M, Rocher V, Moilleron R, Chebbo G (2012): Priority pollutants in urban stormwater: Part 2 - Case of combined sewers. Water Research 46, 6693-6703.

Gasperi, C. Sebastian, V. Ruban, S. Percot, L. Wiest, C. Mirande, E. Caupos, D. Demare, M. Diallo Kessoo, M. Saad, J. Schwartz, P. Dubois, C. Fratta, H. Wolff, R. Moilleron, G. Chebbo, C. Cren, M. Millet, S. Barraud, MC. Gromaire (2014): Micropollutants in urban stormwater: occurrence, concentrations, and atmospheric contributions for a wide range of contaminants in three French catchments.. Environmental Science and Pollution Research, 1-15.

Gilbert S, Gasperi J, Rocher V, Lorgeoux C, Chebbo G (2012): Removal of alkylphenols and polybromodiphenylethers by a biofiltration treatment plant during dry and wet-weather periods. Water Science and Technology 65, 1591-1598.

Gromaire M.-C. (1998) : "La pollution des eaux pluviales urbaines en réseau d'assainissement unitaire : origines et caractéristiques." Thèse de doctorat, Sciences et Technique de l'Environnement, École nationale des Ponts et Chaussées, 507 p.

Gromaire-Mertz MC, Garnaud S, Gonzalez A, Chebbo G (1999): Characterisation of urban runoff pollution in Paris. Water Science and Technology 39, 1-8.

Hites RA (2004): Polybrominated diphenyl ethers in the environment and in people: A meta-analysis of concentrations. Environmental Science \& Technology 38, 945-956.

Huang XJ, Pedersen T, Fischer M, White R, Young TM (2004): Herbicide runoff along highways. 1. Field observations. Environmental Science \& Technology 38, 3263-3271.

Kalmykova Y, Bjorklund K, Stromvall A-M, Blom L (2013): Partitioning of polycyclic aromatic hydrocarbons, alkylphenols, bisphenol A and phthalates in landfill leachates and stormwater. Water Research 47, 1317-28.

Kolpin DW, Thurman EM, Lee EA, Meyer MT, Furlong ET, Glassmeyer ST (2006): Urban contributions of glyphosate and its degradate AMPA to streams in the United States. Science of the Total Environment 354, 191-197.

Lamprea K, Ruban V (2011a): Pollutant concentrations and fluxes in both stormwater and wastewater at the outlet of two urban watersheds in Nantes (France). Urban Water Journal 8, 219231.

Lamprea K, Ruban V (2011b): Characterization of atmospheric deposition and runoff water in a small suburban catchment. Environmental Technology 32, 1141-1149.

Metadier M, Bertrand-Krajewski JL (2012): The use of long-term on-line turbidity measurements for the calculation of urban stormwater pollutant concentrations, loads, pollutographs and intraevent fluxes. Water Research 46, 6836-6856.

Motelay-Massei A, Garban B, Phagne-Larcher K, Chevreuil M, Ollivon D (2006): Mass balance for polycyclic aromatic hydrocarbons in the urban watershed of Le Havre (France): Transport and fate of PAHs from the atmosphere to the outlet. Water Research 40, 1995-2006.

Muller K, Bach M, Hartmann H, Spiteller M, Frede HG (2002): Point- and nonpoint-source pesticide contamination in the Zwester Ohm catchment, Germany. J. Environ. Qual. 31, 309-318.

Muresan B, Lorgeoux C, Gasperi J, Moilleron R (2010): Fate and spatial variations of polybrominated diphenyl ethers in the deposition within a heavily urbanized area: Case of Paris (France). Water Science and Technology 62, 822-828.

Percot S 2012: Contributions des retombées atmophériques aux flux de polluants issus d'un petit bassin versan urbain - Cas du Pin sec à Nantes, Ecole centrale de Nantes, 210 pp.

Pitt R, Field R, Lalor M, Brown M (1995): Urban stormwater toxic pollutants - Assessement, sources and treatability. Water Environment Research 67, 260-275.

Pitt R, Maestre A (2005): Stormwater quality as described in the National Stormwater Quality Database (NSQD). 10th International Conference on Urban Drainage, Copenhagen/Denmark Quaghebeur D, De Smet B, De Wulf E, Steurbaut W (2004): Pesticides in rainwater in Flanders, Belgium: results from the monitoring program 1997-2001. Journal of Environmental Monitoring 6, 182-190.

Rossi L 1998: Qualité des eaux de ruissellement urbaines. PhD Thesis Thesis, Ecole Polytechnique Fédérale de Lausanne, Suisse, 303 pp.

Sabin LD, Lim JH, Stolzenbach KD, Schiff KC (2005): Contribution of trace metals from atmospheric deposition to stormwater runoff in a small impervious urban catchment. Water Research 39, 3929-3937.

Saget A 1994: Base de données sur la qualité des rejets urbains de temps de pluie: distribution de la pollution rejetée, dimensions des ouvrages d'interception. PhD Thesis Thesis, Ecole Nationale des Ponts et Chaussées, France, 227 pp. 
Schummer C, Tuduri L, Briand O, Appenzeller BM, Millet M (2012): Application of XAD-2 resinbased passive samplers and SPME-GC-MS/MS analysis for the monitoring of spatial and temporal variations of atmospheric pesticides in Luxembourg. Environmental Pollution 170, 88-94.

Soclo HH, Garrigues P, Ewald M (2000): Origin of polycyclic aromatic hydrocarbons (PAHs) in coastal marin sediments: case studies in Cotonou (benin) and Aquitaine (France) areas. Marine Pollution Bulletin 40, 387-396.

Staples CA, Dorn PB, Klecka GM, O'Block ST, Harris LR (1998): A review of the environmental fate, effects, and exposures of bisphenol A. Chemosphere 36, 2149-2173.

Smullen JT, Shallcross AL, Cave KA (1999): Updating the US nationwide urban runoff quality data base. Water Science and Technology 39, 9-16.

Sternbeck J, Sjodin A, Andreasson K (2002): Metal emissions from road traffic and the influence of resuspension-results from two tunnel studies. Atmospheric Environment 36, 4735-4744.

ter Schure AFH, Larsson P, Agrell C, Boon JP (2004): Atmospheric transport of polybrominated diphenyl ethers and polychlorinated biphenyls to the Baltic sea. Environmental Science \& Technology 38, 1282-1287.

Thorpe A, Harrison RM (2008): Sources and properties of non-exhaust particulate matter from road traffic: A review. Science of the Total Environment 400, 270-282.

Tlili K, Labadie P, Alliot F, Bourges C, Desportes A, Chevreuil M (2012): Polybrominated Diphenyl Ether Dynamics in Ambient Air and Atmospheric Bulk/Wet Deposition in Downtown Paris (France). Water Air and Soil Pollution 223, 1543-1553.

US-EPA 1989: A Probabilistic Methodology for Analyzing Water Quality Effects of Urban Runoff on Rivers and Streams, U.S. Environmental Protection Agency, Office of water, Washington D.C.128.

Ying GG, Williams B, Kookana R (2002): Environmental fate of alkylphenols and alkylphenol ethoxylates - a review. Environment International 28, 215-226.

Yunker MB, Macdonald RW, Goyette D, Paton DW, Fowler BR, Sullivan D, Boyd J (1999): Natural and anthropogenic inputs of hydrocarbons to the Strait of Georgia. Science of the Total Environment 225, 181-209.

Yunker MB, Macdonald RW, Vingarzan R, Mitchell RH, Goyette D, Sylvestre S (2002): PAHs in the Fraser River basin: a critical appraisal of PAH ratios as indicators of PAH source and composition. Organic Geochemistry 33, 489-515.

Zgheib S, Moilleron R, Chebbo G (2011a): Influence of the land use pattern on the concentrations and fluxes of priority pollutants in urban stormwater. Water Science and Technology 64, 1450-1458. Zgheib S, Moilleron R, Saad M, Chebbo G (2011b): Partition of pollution between dissolved and particulate phases: What about emerging substances in urban stormwater catchments? Water Research 45, 913-925.

Zgheib S, Moilleron R, Chebbo G (2012): Priority pollutants in urban stormwater: Part 1-Case of separate storm sewers. Water Research 46, 6683-6692. 


\title{
TITLE
}

Micropollutant in stormwater: findings of the INOGEV research programm

\begin{abstract}
This study aimed at i) providing for a large range of pollutants $(n=77)$ information on their occurrence and concentration ranges in stormwater, ii) assessing the land use influence on stormwater quality and iii) analyzing the contribution of total atmospheric fallout (TAF) and endogenous sources to this contamination. Additionally, for poorly documented pollutants such as nonylphenol and octylphenol ethoxylates (NPnEO and OPnEO), this study reported for the first time levels for some new concerning metals (As, Ti, Cr, V) and organic pollutants such as polybromodiphenylethers (PBDEs), some pesticides and bisphenol A (BPA). For most of the pollutants, the stormwater quality at the outlet of three urban catchments exhibiting a different land use patterns did not significantly differ, suggesting that land use does no significantly influence the stormwater micropollutant contamination. No significant difference in terms of partitioning between all sites was also observed, indicating the not site-dependence. Finally, the contributions of TAF to stormwater contamination for micropollutants were rather low or very low. This underlines not only for PAHs as previously demonstrated in the literature but also for a larger range of molecules such as BPA, NPnEO, OPnEO and PBDEs a high local production related to the leaching of urban surfaces, buildings and vehicles. Knowledge gained in this study may be used soon to elaborate relevant decision support tools for i) urban stormwater practitioners and/or ii) watershed managers to reduce stormwater pollutant discharges
\end{abstract}

